\title{
Balkanologie
}

Balkanologie Revue d'études pluridisciplinaires

Vol. XII, $n^{\circ} 2 \mid 2010$

Volume XII Numéro 2

\section{Les nouvelles migrations dans l'Europe : chaînes migratoires, établissement et réseaux sociaux des Bulgares en Espagne et en Catalogne}

Sílvia Gómez-Mestres et Jose Luis Molina

\section{(2) OpenEdition}

Journals

Édition électronique

URL : http://journals.openedition.org/balkanologie/2211

DOI : 10.4000/balkanologie.2211

ISSN : 1965-0582

Éditeur

Association française d'études sur les Balkans (Afebalk)

Référence électronique

Sílvia Gómez-Mestres et Jose Luis Molina, « Les nouvelles migrations dans l'Europe : chaînes migratoires, établissement et réseaux sociaux des Bulgares en Espagne et en Catalogne »,

Balkanologie [En ligne], Vol. XII, n² 2 | 2010, mis en ligne le 24 janvier 2011, consulté le 17 décembre 2020. URL : http://journals.openedition.org/balkanologie/2211 ; DOI : https://doi.org/10.4000/

balkanologie. 2211

Ce document a été généré automatiquement le 17 décembre 2020.

(c) Tous droits réservés 


\section{Les nouvelles migrations dans l'Europe : chaînes migratoires, établissement et réseaux sociaux des Bulgares en Espagne et en Catalogne}

Sílvia Gómez-Mestres et Jose Luis Molina

\section{Introduction}

\section{À la recherche d'une " nouvelle vie » : les migrations bulgares du XXİ̀me siècle}

1 L'immigrant bulgare quitte son pays à la recherche d'une "vie meilleure " et de nouvelles opportunités, motifs qui comportent des aspects autant personnels que sociaux, économiques, professionnels et politiques. Yordan, un transporteur, le manifeste très clairement : selon lui en Bulgarie, « les lois travaillent pour les pauvres et non pas pour les voleurs $»^{1}$ (c'est-à-dire que les lois existent pour les pauvres et non pour les voleurs, qui bénéficient d'une immunité face à elle - Yordan parle ici de la corruption du pays). Selon Daniela, qui s'est installée il y a quelques années en Espagne et gagne sa vie comme vendeuse ambulante dans les rues de Barcelone : «maintenant je peux dire que j'ai une voiture, un ordinateur, et bon... on est dans un niveau normal, je ne peux pas dire qu'on soit riche mais que je ne peux pas dire non plus que je n'ai pas d'argent pour la nourriture...bon, une vie normale $»^{2}$.

2 Bien que l'on puisse dire que les mouvements migratoires sont, en partie, liés à la crise économique vécue par la Bulgarie après la chute du communisme, et, par conséquent, à l'augmentation concomitante du taux de chômage ainsi qu'à la restructuration du 
marché du travail, on a pu constater que ce ne sont pas les seuls aspects qui ont motivé les migrants bulgares à quitter leur pays ${ }^{3}$.

3 La plupart des migrants interviewés avaient auparavant un travail en Bulgarie et certains jouissaient même d'une bonne position sociale. Leurs motivations étaient surtout la recherche d'une nouvelle vie, de meilleurs revenus, d'un niveau de consommation «normal » semblable à celui des pays occidentaux, et le désir de se développer dans un milieu social prévisible et plus sûr. Pour les étudiants, partir étudier à l'étranger est une question de prestige, un investissement pour leur futur. En définitive, c'est l'envie de changer de vie et de statut qui a motivé les Bulgares à quitter leur pays, en parallèle de motifs économiques.

On ne peut pas ignorer la forte crise économique que la Bulgarie a vécue au début des années 90 , suite aux transformations qui ont accompagné la chute du régime communiste en $1989^{4}$. Cette crise a notamment été marquée par le passage d'une économie planifiée et centralisée à une économie de marché plus libéralisée, avec des conséquences sociales qui ont contribué à créer d'importantes chaînes migratoires et des réseaux sociaux très solides de mobilité vers l'étranger. Mais ce sont surtout l'absence de travail et les bas salaires ainsi que la dégradation du système de sécurité sociale et les fortes inégalités sociales qui ont le plus affecté la société bulgare. Bien que ces aspects n'aient pas affecté la vie professionnelle de tous, ils ont déstabilisé la société et l'ont laissée sans claire perspective d'avenir. Ils s'ajoutent à l'existence de fortes chaînes migratoires pour expliquer le phénomène migratoire des Bulgares à l'étranger et leur arrivée dans des pays comme l'Espagne.

5 Ainsi, on peut dire que les facteurs économiques ne sont pas suffisants pour expliquer les dimensions acquises par le phénomène migratoire à l'époque actuelle et l'on doit recourir, parmi d'autres variables, au rôle clé des réseaux sociaux pour expliquer, par exemple, le moment où est prise la décision d'émigrer et la destination choisie. L'existence de contacts personnels sur le lieu de destination permet de diminuer ce que l'on appelle les dépenses de base de l'émigration: la recherche d'un travail ou d'un logement, l'insertion des nouveaux venus, le maintien des contacts avec la Bulgarie, etc. Les réseaux sociaux aident aussi à expliquer, à travers l'analyse de leur structure et de leur composition, les processus d'adaptation dans la société d'accueil, le changement culturel et l'identification ethnique sur le lieu de destination.

Cette étude analyse la dimension des mouvements migratoires des migrants bulgares en Catalogne (dans le nord-est de l'Espagne) à travers leurs réseaux sociaux. Nous avons examiné comment et pourquoi des Bulgares décident d'immigrer en Catalogne, c'est-à-dire la construction de leurs chaînes migratoires vers cette région de l'Espagne. En outre, nous avons identifié la structure et la composition de leurs réseaux sociaux afin de découvrir les diverses modalités et stratégies de leur adaptation à la société d'accueil, c'est-à-dire que nous avons examiné les différentes façons dont ils essaient d'acquérir le bien-être social à travers l'utilisation des réseaux sociaux, selon leurs possibilités de développement dans le pays de destination. En outre, nous avons évalué la présence et la construction des communautés de migrants bulgares en Catalogne ainsi que le degré de participation des citoyens en observant les associations bulgares existantes. Finalement, nous avons observé les différents schémas d'identification ethnique et culturelle. L'étude est fondée sur un échantillon de 50 personnes (de Barcelone et de Roses) qui ont été interviewées en utilisant la technique de la "boule de neige". Chaque Ego (personne interviewée) désignait 30 Alters (contacts actifs) en 
réponse à la formule suivante : « citez-nous le prénom d'environ 30 personnes que vous connaissez, avec qui vous avez eu un contact ces deux dernières années au moins et avec qui vous pourriez communiquer encore une fois si nécessaire. Il est important que toutes les catégories de contacts (famille, amis, collègues ...) soient représentées ».

Les données ont été recueillies à l'aide d'EgoNet, un programme ouvert pour le recueil des données des réseaux sociaux. Il permet la visualisation des réseaux personnels et analyse en profondeur le sens donné par l'informateur à son monde social (Ego n'est pas dans le réseau social). Les différentes variables recueillies pendant l'interview peuvent être converties en variables visuelles et combinées de différentes manières afin qu'il soit possible pour l'intervieweur et pour l'informateur d'étudier conjointement et de découvrir de nouvelles idées sur le réseau de contacts personnels. En outre, pendant le processus de recueil de données, un vaste travail sur le terrain interviews semi-structurées et entretiens approfondis avec des informateurs clés - a été effectué, ce qui nous a fourni des informations de qualité sur les sujets d'intérêt concernant cette recherche (identité et identification, manifestations culturelles, associations de Bulgares).

\section{Un bref regard historique sur les mobilités bulgares $(1900-2010)$}

8 Bien que l'étude des processus de migration a connu un très vif intérêt parmi les recherches et travaux récents qui concernent les Bulgares et la Bulgarie post-socialiste, les processus migratoires en Bulgarie ne sont pas nouveaux 5 . Après 1878 - c'est-à-dire après la sortie de l'empire ottoman, des mouvements migratoires massifs ont été causés par des accords bilatéraux entre divers États balkaniques: la Serbie, la Bulgarie, la Grèce, la Roumanie et la Turquie ont échangé certains segments de leurs populations. Il s'agissait de mouvements ethniques. Plus tard, en 1930, un autre mouvement de population, coïncidant avec la crise mondiale, a pris la direction des États Unis ${ }^{6}$ et du Canada, mais aussi d'autres pays anciennement provinces austro-hongroises, renforçant la diaspora bulgare en Roumanie, Autriche, Hongrie, République Tchèque, Slovaquie, Pologne, Ukraine. Il s'agissait de migrations clairement motivées par des raisons économiques et professionnelles.

9 Avec le régime communiste (1944-1989), le mouvement transnational a été théoriquement interrompu, car seules les personnes ayant un permis spécial pouvaient voyager. Malgré tout, il y a eu beaucoup de mouvements pendant cette période. Il s'agissait surtout d'émigrations ethniques, de Juifs-bulgares qui partaient vers Israël, d'Arméniens bulgares vers l'Arménie, et aussi de Turcs-Bulgares vers la Turquie. Pour la plupart, ces migrations ont été motivées par la collectivisation des terres en 1946 : une première vague a eu lieu en 1950-1951, et une seconde s'est produite plus tard, entre 1968-1978, conséquence de la réunification familiale. Mais ceux qui ne partageaient pas l'idéologie des dirigeants politiques communistes du pays partirent eux aussi, devenant des migrants politiques, certes en très petit nombre. Autour de la fin des années 1940, ils s'établissaient majoritairement aux États-Unis.

10 Au début de la période post-communiste, à partir de 1989, période marquée par la libéralisation des passeports et l'abolition des conditions pour l'obtention du visa, la sortie massive des Turcs-Bulgares est motivée par la politique d'homogénéisation ethnique qui depuis 1985 provoquait la servitude de la population d'origine turque. 
L'opération consistait à faire disparaitre les noms et prénoms turcs en les substituant par des noms bulgares-slaves, même avec des effets rétroactifs, sur les parents décédés dans les deux dernières générations. Plusieurs mosquées ont été brûlées et on a interdit aux médecins turcs de pratiquer les circoncisions. Officiellement, on a dit que cette minorité turque (et turcophone) n'était constituée que par des Bulgares qui s'étaient convertis à l'islam pendant la domination ottomane. Pour ajouter à la confusion, cependant, on y comptait aussi les Pomaks, Bulgares de religion musulmane mais de langue slave. Tout ce processus a obligé l'émigration / le retour de nombreux BulgaresTurcs (pendant l'été 1989 à peu près 300000 partent en Turquie) ${ }^{7}$.

11 En même temps, c'est lors de cette première vague migratoire - entre 1990 et 2001 qu'ont été tracés les chemins des premiers départs de Bulgares vers la Grèce, l'Italie, les Pays-Bas, l'Allemagne, pays plus ou moins voisins, mais aussi vers des pays où la présence des Bulgares est déjà une tradition historique (États-Unis et Canada). L’arrivée des Bulgares en Espagne est plus tardive; jusqu'au milieu des années 1990, leur présence y est inconnue ${ }^{8}$. Mais avec l'entrée de la Bulgarie dans l'espace Schengen, à partir de 2001, les ressortissants bulgares peuvent rester dans n'importe quel pays Schengen sans visa, pour une période de trois mois renouvelable tous les six mois à partir du premier jour d'entrée dans la zone Schengen. C'est ce qui explique l'entrée massive de Bulgares en Espagne jusqu'en 2006. Bien qu'au début ce pays n'ait pas été leur destination favorite, leur présence de plus en plus constante et importante ces dernières années a converti l'Espagne en une zone de mobilité des citoyens bulgares parmi les plus importantes de la Méditerranée9 .

12 C'est surtout à partir du $1^{\text {er }}$ janvier 2007, date de l'entrée de la Bulgarie et de la Roumanie dans l'Union Européenne, que la présence bulgare en Espagne se renforce: régularisation des illégaux mais aussi nouvelles entrées. Cette situation a contribué à mieux visualiser une collectivité jusqu'alors très invisible. Les accroissements de population les plus importants, en pourcentages et selon les nationalités, ont été celui des citoyens roumains ( +20000 individus, $24,98 \%$ du total des immigrants) et celui des Bulgares (15,10\%).

13 En septembre $2007^{10}, 37,30 \%$ des étrangers venaient de l'UE (y compris Bulgares et Roumains). Dans cette proportion, 3,04\% correspondent au collectif bulgare (113 792 individus), occupant ainsi la huitième position d'un classement où les Roumains sont en deuxième position (505 670 individus) ${ }^{11}$. Le 30 juin 2008, la somme des deux collectifs migrants représentait déjà $49,31 \%$ du total des citoyens communautaires résidant en Espagne ${ }^{12}$. Le gouvernement espagnol a appliqué dès le $1^{\mathrm{er}}$ janvier 2007 un moratoire sur la libre circulation des travailleurs roumains et bulgares, fixant que sur une période de deux ans, ceux-ci ne pourraient pas travailler librement en Espagne à moins d'avoir un permis de résidence ${ }^{13}$. En 2009, l'échéance de ce moratoire a favorisé l'établissement définitif des familles et groupes bulgares.

Évolution en chiffres et pourcentage de la présence de la population bulgare dans la population migrante qui réside en Espagne et par comparaison avec la population roumaine depuis 2006 jusqu'à 2010 selon les rapports annuels

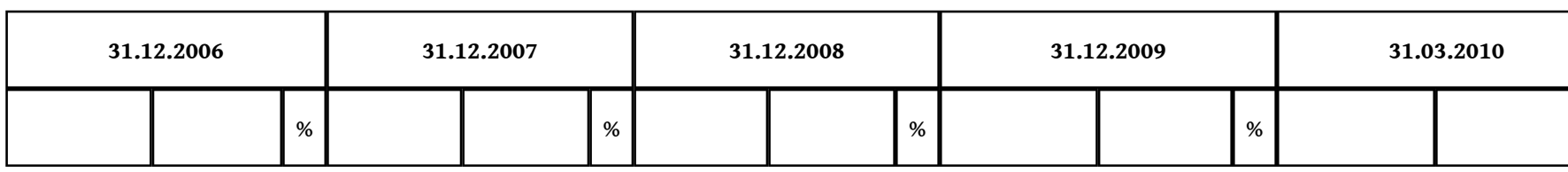




\begin{tabular}{|c|c|c|c|c|c|c|c|c|c|c|c|c|c|}
\hline Maroc & 543721 & 18 & Maroc & 648735 & 16 & Maroc & 717416 & 16 & Maroc & 767784 & 16 & Maroc & 775054 \\
\hline Equateur & 376233 & 12 & Roumanie & 603889 & 15 & Roumanie & 718844 & 16 & Roumanie & 751688 & 16 & Roumanie & 772137 \\
\hline Colombie & 225504 & 7 & Equateur & 395808 & 10 & Equateur & 421527 & 9 & Equateur & 440304 & 9 & Equateur & 437279 \\
\hline Roumanie & 211325 & 7 & Colombie & 254301 & 6 & Colombie & 274832 & 6 & Colombie & 287205 & 6 & Colombie & 284940 \\
\hline $\begin{array}{c}\text { G. } \\
\text { Bretagne }\end{array}$ & 175870 & 6 & $\begin{array}{l}\text { G. } \\
\text { Bretagne }\end{array}$ & 198638 & 5 & G.Bretagne & 219738 & 5 & G.Bretagne & 222039 & 5 & G.Bretagne & 223213 \\
\hline Chine & 99526 & 3 & Bulgarie & 127058 & 3 & Bulgarie & 144401 & 3 & Chine & 151547 & 3 & Italie & 160967 \\
\hline Italie & 98481 & 3 & Italie & 124936 & 3 & Italie & 139132 & 3 & Italie & 150667 & 3 & Chine & 154056 \\
\hline Pérou & 90906 & 3 & Chinois & 119859 & 3 & Chine & 138558 & 3 & Bulgarie & 147080 & 3 & Bulgarie & 150930 \\
\hline Argentine & 86921 & 3 & Pérou & 116202 & 3 & Pérou & 130900 & 3 & Pérou & 144620 & 3 & Pérou & 143712 \\
\hline Allemagne & 77390 & 3 & Portugal & 101818 & 3 & Portugal & 121918 & 3 & Portugal & 126928 & 3 & Portugal & 128970 \\
\hline Portugal & 72505 & 2 & Argentine & 96.056 & 2 & Allemagne & 102202 & 2 & Bolivie & 117106 & 2 & Bolivie & 121991 \\
\hline Bulgarie & 60174 & 2 & Allemagne & 91670 & 2 & Argentine & 97277 & 2 & Allemagne & 109438 & 2 & Allemagne & 112236 \\
\hline Autres & 903252 & 31 & Autres & 1100044 & 29 & Autres & 1246754 & 29 & Autres & 1374826 & 29 & Autres & 1377014 \\
\hline Total & 3021808 & & Total & 3979014 & & Total & 4473499 & & Total & 4791232 & & Total & 4842499 \\
\hline
\end{tabular}

Source : d'après Ministère de l'Intérieur, statistiques sur l'immigration (http://extrangeros.mtin.es/es/ informacionEstadistica)

\section{Les Bulgares en Espagne et en Catalogne}

14 Les motifs qui amènent les Bulgares en Espagne ne sont pas dus au hasard. Dans un premier temps, l'entrée des Bulgares en Espagne a été motivée par des accords de recrutement de travail antérieurs à l'entrée de la Bulgarie et de la Roumanie dans l'UE. À partir du moment où ces pays en sont devenus membres, l'entrée contingentée n'a plus été applicable. Entre 2007 et 2009, les Bulgares entrent donc en suivant la voie d'entrée commune : le circuit d'entreprise. En d'autres termes, ils doivent contacter par eux-mêmes l'entreprise qui pourrait les embaucher, soit parce qu'ils la connaissent à la suite d'un séjour de travail, soit parce qu'ils ont répondu à des demandes trouvées, par exemple, sur le web, et cela sans connaissance préalable de l'entreprise ni de l'entrepreneur qui les embauche. En règle générale, les Bulgares travaillent en petite et moyenne entreprise, dans les secteurs de la construction, du transport et du métal ${ }^{14}$. Certains travaillent aussi dans les services aux particuliers (nettoyages, tâches agricoles saisonnières, hôtellerie et catering). Un petit nombre ouvre des petites entreprises : surtout bars et restaurants, mais aussi entreprises d'importation de produits bulgares et artisanaux ${ }^{15}$. 
15 Il faut cependant signaler qu'une part non négligeable d'entre eux a une formation universitaire, exerce une profession libérale (ils sont musiciens, artistes, etc.), ou bien encore a l'idée de terminer leurs études. C'est ce que l'on constate déjà parmi les premiers Bulgares qui arrivent en Espagne, durant la seconde moitié des années $90^{16}$.

Les Bulgares qui ont travaillé au préalable en Espagne ont déjà une connaissance du pays et de la langue, ce qui facilite le contact avec les entreprises et les entrepreneurs. Ils font alors office d'intermédiaires pour attirer leurs compatriotes, ce qui aboutit à la création de différentes chaînes migratoires différentes construites sur des réseaux de famille, de parenté, d'amitié et de voisinage ${ }^{17}$, voire de caractère ethnique ${ }^{18}$. De plus, les avantages légaux de la libre circulation et du travail, obtenus par l'entrée dans l'UE, ont facilité l'installation des immigrés en Catalogne et ouvert la voie, durant ces deux dernières années, au regroupement familial.

À côté de ces avantages légaux, le marché du travail de l'Europe méridionale exerce une certaine attraction sur les migrants venus de l'Europe du Sud-Est, y compris les Bulgares, par son dynamisme et ses exigences minimes, requérant une main d'œuvre peu qualifiée. L'importante demande de travail saisonnier dans les villages de la côte catalane a ainsi permis l'entrée de plusieurs groupes de migrants vers la Catalogne. La relative tolérance envers les travailleurs sans papiers, aussi bien de la part des autorités espagnoles que chez les entrepreneurs, a fait de l'Espagne une destination prisée pour ces migrants ${ }^{19}$, à quoi il faut ajouter le climat, le paysage, mais aussi la proximité culturelle que ceux-ci déclarent ressentir avec l'Espagne. D'ailleurs, dans les pays du Sud-est de l'Europe, l'image de l'Espagne et du mode de vie des Espagnols a inévitablement influencé la direction que prennent les flux migratoires de beaucoup de Bulgares mais aussi des Roumains.

Les interviewés racontent que le choix de l'Espagne a d'abord été motivé par leurs contacts dans ce pays (c'est-à-dire par les réseaux sociaux), mais aussi par le fait qu'ils connaissaient déjà le pays, qu'ils y étaient venus y passer leurs vacances et faire du tourisme. D'autres personnes, sans être venues au préalable, manifestent qu'elles ressentent une certaine attraction pour l'Espagne et concrètement pour Barcelone (bien qu'en règle générale la première ville où elles se soient installées ait été Madrid parce qu'il s'agit de la capitale) ou bien d'autres villes côtières, selon leurs contacts sur le lieu de destination et selon la demande de travail.

19 Leur arrivée n'a été pas continue et leur répartition sur le territoire n'est pas non plus homogène. Les principales villes d'accueil sont Madrid (22 585) dans la communauté de Madrid $^{20}$, Valence (17 751) et Alicante (9 271) dans la communauté valencienne, la communauté des îles Baléares (8 160), enfin Valladolid (7 545) dans la communauté de Castille-et-León. Leur présence sur le territoire catalan est plus faible : en 2008, on n'y compte que 11142 Bulgares, dont 5486 habitent dans la province de Barcelone, 1776 dans celle de Girone, 1856 à Lérida et 2024 à Tarragone ${ }^{21}$. La Catalogne ne vient ainsi qu'en cinquième position dans le classement des préférences des Bulgares.

20 Ces différences dans la distribution territoriale s'expliquent d'abord par la diversité des chaînes migratoires: certaines favorisent les grandes concentrations dans quelques villes, d'autres l'établissement disséminé et dispersé dans certaines parties du territoire espagnol, d'autres encore les deux, ce qui a une incidence sur l'existence ou non d'établissements ethniques. Les caractéristiques particulières au lieu d'accueil expliquent également l'absence d'homogénéité dans la répartition spatiale : petite ou grande ville, structure de population, organisation locale des relations humaines. Tous 
ces aspects peuvent laisser leur trace dans la composition et la structure des réseaux sociaux des migrants qui doivent reconstruire leurs liens personnels, et se traduisent par des différences que ce soit dans les formes basiques de changement culturel ou dans l'identification ethnique des communautés de migrants.

Présence des Bulgares sur le territoire espagnol dans les villes où ils sont le plus nombreux (en gras, chiffres remarquables)

\begin{tabular}{|c|c|c|}
\hline Communauté & Ville & Bulgares \\
\hline ANDALOUSIE & & 10454 \\
\hline ARAGON & & 6172 \\
\hline ASTURIES & & 514 \\
\hline ÎLES BALÉARES & & 8160 \\
\hline CASTILLE-LA MANCHE & & 7063 \\
\hline \multirow{9}{*}{ CASTILLE-et-LEÓN } & Avila & 840 \\
\hline & Burgos & 5181 \\
\hline & León & 2690 \\
\hline & Palencia & 1083 \\
\hline & Salamanq & que 1111 \\
\hline & Ségovie & 7420 \\
\hline & Soria & 1431 \\
\hline & Valladoli & id 7545 \\
\hline & Zamora & 1727 \\
\hline Total & & 29028 \\
\hline \multirow{3}{*}{ VALENCIENNE } & Alicante & 9271 \\
\hline & Castellon & 808 \\
\hline & Valence & 17751 \\
\hline Total & & 27830 \\
\hline ESTRÉMADURE & & 428 \\
\hline GALICE & & 767 \\
\hline
\end{tabular}




\begin{tabular}{|l|l|c|}
\hline MADRID & & $\mathbf{2 2} \mathbf{5 8 5}$ \\
\hline \multirow{2}{*}{ MURCIE } & & 6460 \\
\hline \multirow{2}{*}{ NAVARRE } & & 7045 \\
\hline \hline \multirow{2}{*}{ PAYS BASQUE } & & 1070 \\
\hline \hline \multirow{2}{*}{ LA RIOJA } & & \multicolumn{2}{|c|}{886} \\
\hline \multirow{2}{*}{ CEUTA } & & \multicolumn{2}{|c|}{1} \\
\hline \hline \multirow{2}{*}{ MELILLA } & & \multicolumn{2}{|c|}{0} \\
\hline \hline \multirow{2}{*}{ Total } & & $\mathbf{1 1} \mathbf{1 4 2}$ \\
\cline { 2 - 4 } & Barcelone & 5486 \\
\cline { 2 - 4 } & Gérone & 1776 \\
\cline { 2 - 4 } & Lérida/Lleida & 1856 \\
\cline { 2 - 4 } & Tarragone & 2024 \\
\hline & & \\
\hline
\end{tabular}

\section{Chaînes migratoires et modèles de mobilité}

L'existence de chaînes migratoires unissant l'Espagne à la Bulgarie n'est pas à prouver. Les Pomaks, des Bulgares de langue bulgare et de confession musulmane, en sont une illustration fameuse. Originaires de Brushlyan, dans les Rhodopes, ils se sont installés à Tafalla, dans les Pyrénées navarraises. Arrivé il y a dix ans déjà, ce groupe, qui réunit aujourd'hui presque 2000 individus, représente un véritable îlot ethnique ${ }^{22}$.

Madrid, Alicante et Valence abritent elles aussi d'importants groupes de Bulgares. Par exemple, dans les petites villes de Getafe et Parla à Madrid, ils ont été nombreux à s'établir, quoique la composition ethnique soit très hétérogène ${ }^{23}$.

Bien que leur présence y ait été timide au début, leur croissance progressive en Catalogne s'explique évidemment par une question de chaîne migratoire. En effet, ce groupe représente la première vague ou, au plus, la seconde, et a été repéré ces cinq ou six dernières années, ce qui permet d'affirmer l'existence de chaînes migratoires. Celles-ci, dans ce cas, sont essentiellement familiales, c'est-à-dire qu'elles se créent avec l'émigration échelonnée dans le temps de toute la famille. Bientôt après, ce groupe initial a été suivi par des amis ou des voisins. La famille et les relations de parenté constituent un lien plus fort même que la communauté ethnique et religieuse, comme on le constate dans le cas des Pomaks en Navarre ${ }^{24}$ ainsi que des Rroms ${ }^{25}$. Ainsi se construisent des réseaux de chaînes familiales et d'amitié indépendantes les unes des autres; la traduction sur le terrain en est que des membres de la même famille, ou des amis et voisins de la famille, s'établissent dans plusieurs localités différentes ${ }^{26}$. Et bien 
que la mobilisation soit familiale, certains viennent individuellement, ou encore en tant que mariées ou fiancées ${ }^{27}$.

Il s'agit pour la plupart de migrants à caractère permanent, c'est-à-dire qui cherchent à s'établir à jamais en Catalogne, tout d'abord avec un contrat à durée déterminée, comme par exemple un contrat saisonnier d'été, et postérieurement en cherchant du travail pour rester sur le lieu de destination. D'autres étaient déjà dans un premier temps en contact sur leur lieu d'origine avec l'entreprise qui les a embauchés, ce qui est alors la conséquence d'un accord d'embauche signé en 2007 entre l'entreprise en Catalogne et la Bulgarie, et ils font ensuite venir leur famille pour rester définitivement sur le lieu de destination. Pour d'autres encore, la perspective est universitaire : une proportion assez grande de migrants est constituée d'étudiants, venus pour poursuivre des études à l'étranger et ayant choisi l'Espagne ${ }^{28}$. Ceux-là arrivent habituellement tous seuls, sans contacts ou presque, mais certains connaissent déjà le pays pour y être venus dans un voyage antérieur. Beaucoup d'entre eux affirment qu'ils veulent rester en Espagne et qu'après leurs études ils y chercheront du travail.

En ce qui concerne l'ethnie à lequel appartiennent les Bulgares qui viennent en Espagne, on peut dire qu'on trouvera très difficilement d'établissements clairement ethniques en Espagne, à l'exception des Pomaks qui résident à Tafalla. Même si les appartenances sont très variées - Turcs, Pomaks, Rroms, Arméniens - en règle générale les groupes cohabitent sans apparente distinction.

Les Rroms bulgares représentent un cas très particulier en Espagne. Leur présence est très discrète, au contraire de Rroms originaires d'autres pays du Sud-est européen, par exemple ceux de Roumanie, bien connus en Catalogne ${ }^{29}$. Selon Marusiakova \& Popov et Ragaru ${ }^{30}$, la migration de ces populations vers l'Europe occidentale diffère grandement selon leur pays d'origine, Bulgarie ou Roumanie. Deux facteurs les informent : leur niveau d'intégration sociale dans leur pays d'origine et la possibilité de retourner à la vie sédentaire pour des raisons économiques. Ainsi, selon Marusiakova \& Popov, si les Bulgares roms sont mieux intégrés dans la population bulgare ${ }^{31}$ que ne le sont leurs congénères en Roumanie, c'est qu'ils se sont sédentarisés et suivent les mêmes schémas migratoires que le reste de la population bulgare, en suivant des chaînes fondées surtout sur la famille comme unité sociale de base, et non sur la communauté ou l'ethnicité. Ce qui caractérise donc les Bulgares en général et les Rroms de Bulgarie, c'est la multiplicité des stades de migration - migrations des parents qui se déroulent en plusieurs étapes -, alors que les Rroms roumains ne migrent généralement pas en familles isolées ou individuellement, mais en communauté.

Il faut tout de même mentionner qu'un des groupes de Rroms bulgares qu'on trouve en Espagne est constitué par les Rudari, qui parlent roumain, ce qui facilite encore plus leur invisibilité et la confusion avec les Rroms roumains ${ }^{32}$.

En Catalogne, on ne connaît pas d'établissements de Rroms bulgares, bien qu'on sache qu'ils existent à Barcelone. Il s'agit d'hommes, venus seuls, sans leur famille, et qui gagnent leur vie en jouant de la musique. Barcelone est la dernière des étapes de leur tour en Europe et elle en représente aussi la fin. La plupart d'entre eux choisissent cette ville comme "seconde résidence ", à côté de leur résidence natale en Bulgarie, habitant ainsi en alternance entre un pays et l'autre, réalisant des séjours saisonniers tout comme s'il s'agissait de gurbet traditionnel ${ }^{33}$. En effet, ce modèle de mobilité alternante (ou pendulaire) ${ }^{34}$, qui avant 2007 semblait conditionné par le renouvellement de visa, perdure actuellement ${ }^{35}$. À Barcelone, ces hommes font partie du cosmopolitisme de 
Barcelonnette, le quartier du port, où ils habitent et trouvent un public de touristes et de ruraux peut-être attirés par l'imaginaire qui s'est créé autour de la musique rrom des Balkans. En regard de l'absence d'études sur les Bulgares en Catalogne, on peut considérer que celles qui ont pour sujet les Rroms bulgares à Barcelone sont nombreuses, et ceci malgré le fait que leur groupe ne soit numériquement pas très important ${ }^{36}$.

29 Pour le reste des Rroms, ils intègrent des réseaux de relations avec d'autres migrants bulgares, sans y occuper de position centrale, de façon périphérique - c'est-à-dire qu'ils utilisent et intègrent les mêmes chaînes migratoires et partagent avec n'importe quel Bulgare arrivant en Espagne les mêmes stratégies d'établissement. De façon générale, on peut trouver, dans la composition des réseaux sociaux bulgares, d'autres Bulgares appartenant à des ethnies différentes. Il est habituel de trouver des familles bulgares turques parmi les contacts des familles d'ethnie bulgare. D'ailleurs, il faut considérer que les Bulgares-turcs sont un des groupes ayant le moins émigré ces dernières années; parmi eux, ceux qui partent vont vers la Turquie ou l'Allemagne, utilisant les chaînes migratoires des Turcs d'autres pays.

\section{Réseaux sociaux des Bulgares en Catalogne}

Les immigrants que nous avons interviewés peuvent être regroupés de la façon suivante : 1 . jeunes ayant terminé leurs études secondaires et quittant la Bulgarie pour mener une vie indépendante ailleurs ; 2 . jeunes étudiants désireux de poursuivre leurs études universitaires ou d'en commencer de nouvelles; 3. travailleurs qualifiés; 4. travailleurs peu qualifiés ; et 5. professions libérales (artistes, artisans, écrivains, etc.).

Les réseaux sociaux des Bulgares sont très influencés par le niveau de formation, l'âge et la voie d'entrée, mais aussi par la structure de la population dans laquelle ils s'incorporent, ou encore par les schémas de relations qui existent dans chaque localité et qui ne sont pas identiques dans les petits villages ou dans les grandes villes. D'autres variables moins évidentes modifient ces réseaux : temps de résidence en destination, acquisition de certaines compétences (connaissance de la langue) et de ressources sociales (connaissance des gens au niveau local, etc.). Ces derniers aspects peuvent être acquis par la participation à des espaces de socialisation de la société d'accueil : espaces institutionnels (écoles, centres civiques, associations) ou espaces professionnels et de loisirs (centres sportifs, bars et célébrations locales).

Les réseaux sociaux des Bulgares sont plutôt liés à la famille, dans laquelle l'unité basique de relation et la plus importante est le cercle fermé des parents, auquel on ajoute les amis (très souvent des amis de la famille), qui dans le contexte de migration acquièrent un statut de quasi-parent. Plus tard viennent s'y ajouter voisins et/ou connaissances. Parmi ces contacts on peut trouver des contacts locaux et internationaux (c'est-à-dire des individus d'autres nationalités qui ne sont ni bulgares ni espagnols). Les contacts s'établissent de façon informelle. On peut dire qu'il s'agit de réseaux sociaux qui s'établissent par des familles qui se rencontrent entre elles en constituant des groups de relation. On peut distinguer trois types de réseaux personnels des migrants bulgares :

a) réseaux très homogènes et denses qui se composent de contacts uniquement bulgares, la plupart d'origine, c'est-à-dire qu'il s'agit de réseaux très transnationaux. En règle générale, les individus qui constituent ce type de réseau sont âgés entre 30 et 40 
ans, ont une famille, se sont installés dans de petites agglomérations catalanes depuis peu (1 à 3 ans). Ils sont arrivés par des chaînes clairement familiales ou de voisinage ; poussés par des raisons professionnelles, ils sont parfois venus travailler dans la même entreprise que ceux qui les ont fait venir. Cependant, quoiqu'en moindre proportion, un certain nombre de personnalités de ces réseaux habitent en grande agglomération; dans ce cas, il s'agit d'individus avec un fort sens d'identification ethnique, cherchant à maintenir cette identification à travers leurs contacts avec des compatriotes; ils travaillent dans de petites entreprises ethniques et n'ont que des clients bulgares; ils sont habituellement invisibles pour la société d'accueil (fig. 1).

Fig. 1. Réseau social de Petranka (femme, 40 ans, mariée), habitant à Roses.

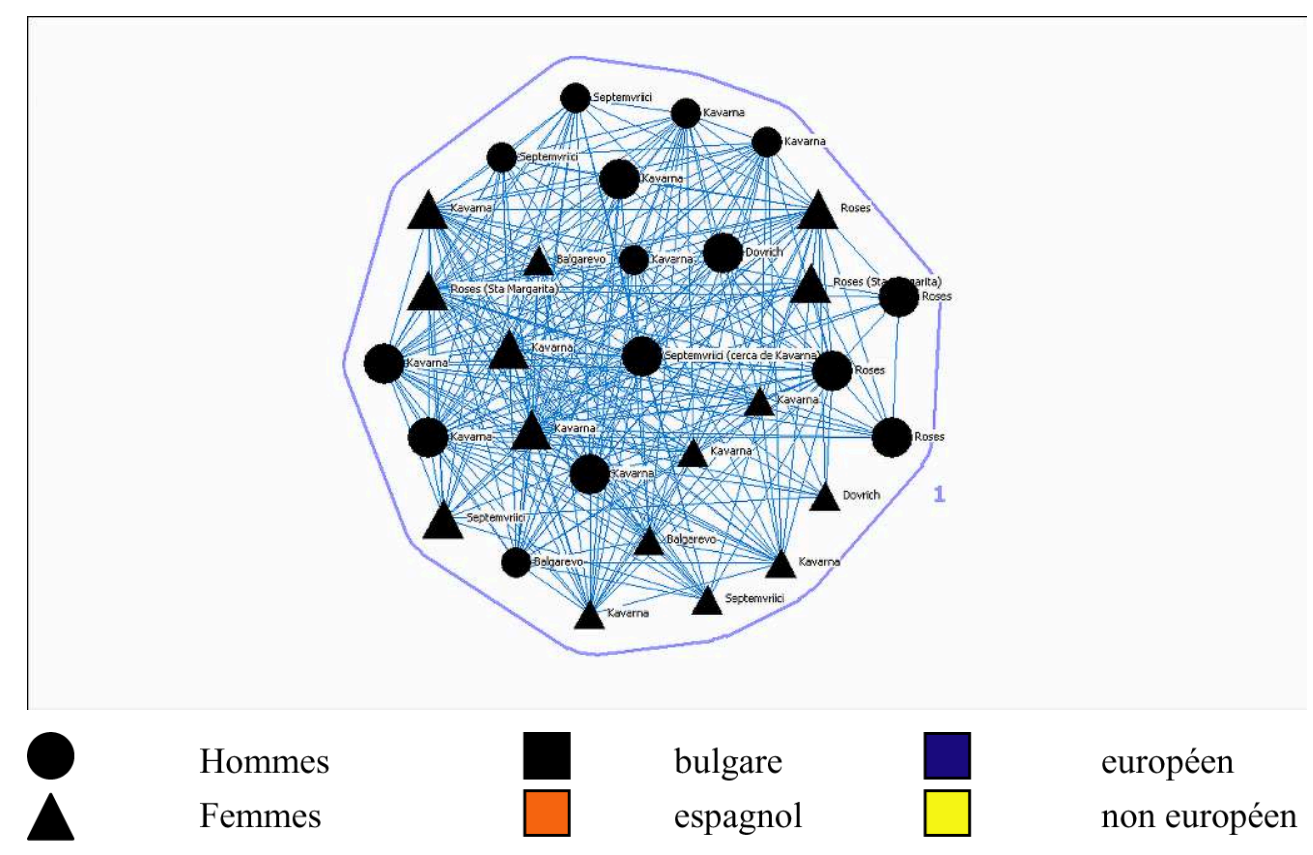

b) réseaux personnels hétérogènes mais assez denses. Les contacts sont très diversifiés, c'est-à-dire avec une considérable proportion d'individus de nationalité autres que bulgare ou espagnole, et aussi avec des contacts locaux (espagnols ou Bulgares résidant en destination). La structure du réseau se distribue entre un ou deux groupes, avec relativement peu de contact sur le lieu d'origine (le réseau n'est pas transnational). Ce type de réseau peut être trouvé dans les petites agglomérations, et correspond à des individus assez jeunes (20-30 ans) venus pour la plupart sans ou presque sans contact mais qui résident déjà en Catalogne depuis assez longtemps (4-5 ans au minimum). Ils maintiennent d'importantes relations avec la population migrante bulgare mais aussi avec la population locale du lieu d'établissement. Souvent, ils servent d'intermédiaire, de pont, entre la collectivité bulgare et la société d'accueil ; leur schéma d'adaptation est dual ou partiel. Ce sont des groupes très visibles pour la société d'accueil ; ils ont clairement changé de culture dans leur vie publique, mais maintiennent leur culture propre en privé (cercle fermé des parents et amis bulgares en destination) (fig. 2) 
Fig. 2. Réseau social de Peslava (femme, 30 ans, célibataire), habitant à Roses.

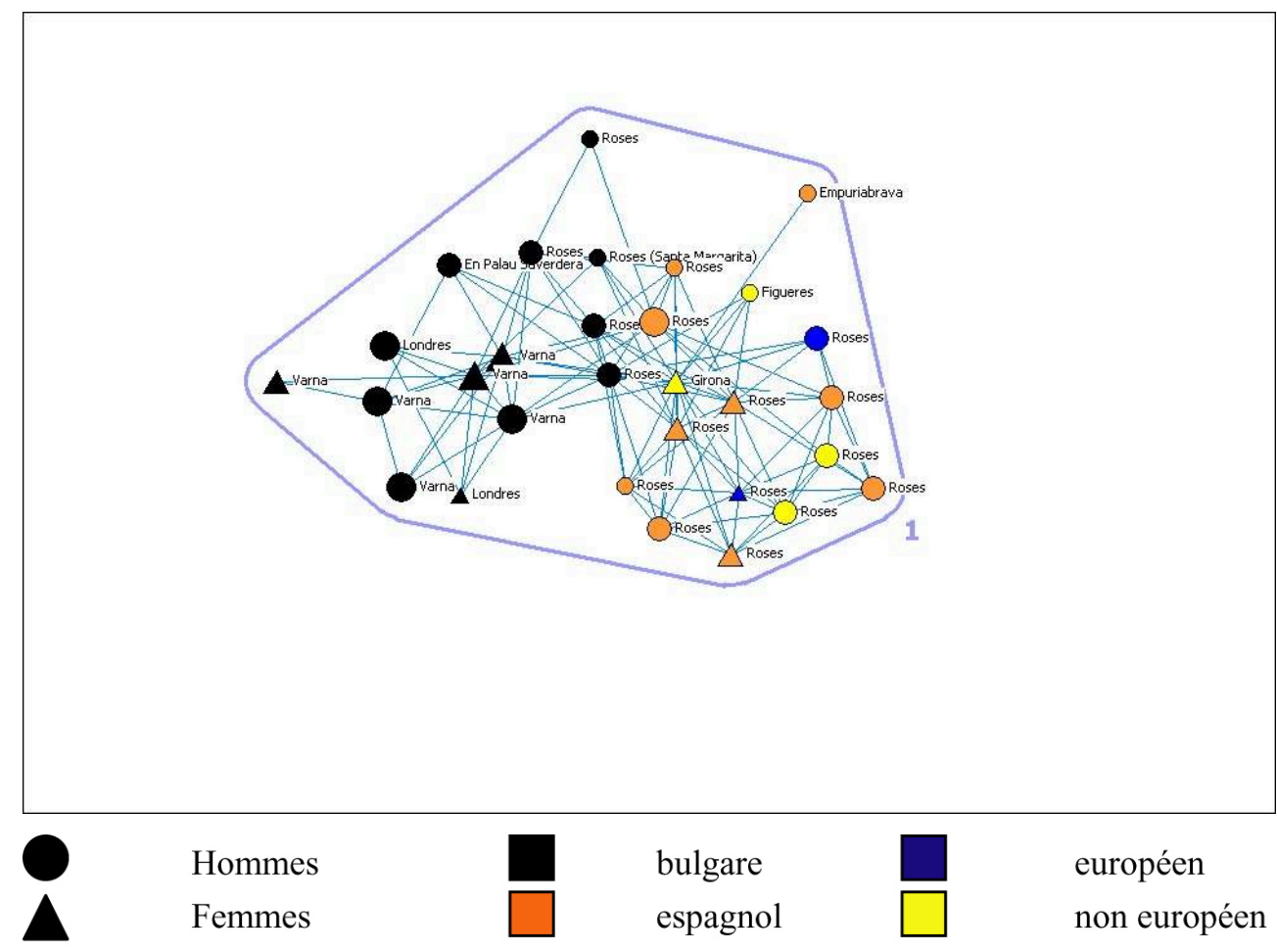

Remarque: la moitié des contacts bulgares se trouve en origine de migration, l'autre en destination.

c) Des réseaux sociaux très hétérogènes, diversifiés et peu denses. Il s'agit de réseaux personnels d'individus habitant en grandes agglomérations, avec une proportion très importante de contacts d'autres nationalités (ni bulgares ni espagnols), mais aussi une proportion assez importante de locaux et quelques Bulgares - normalement la petite famille qui réside en origine. Dans les grandes villes, la dispersion de la population dans les différents voisinages est très notable. Pour autant, les relations sociales ne sont pas aussi denses ni intenses que dans les petites agglomérations. L'âge de ces individus oscille entre 20 et 50 ans; venus avec peu de contacts en destination, ils viennent pour la plupart afin de terminer leurs études, de chercher du travail - certains d'entre eux essaient d'ouvrir de petites entreprises. Globalement, on peut dire qu'il s'agit d'un groupe très invisible (voire fig. 3 ) 
Fig. 3. Réseau social de Vesela (femme, 32 ans, célibataire), habitant à Barcelone

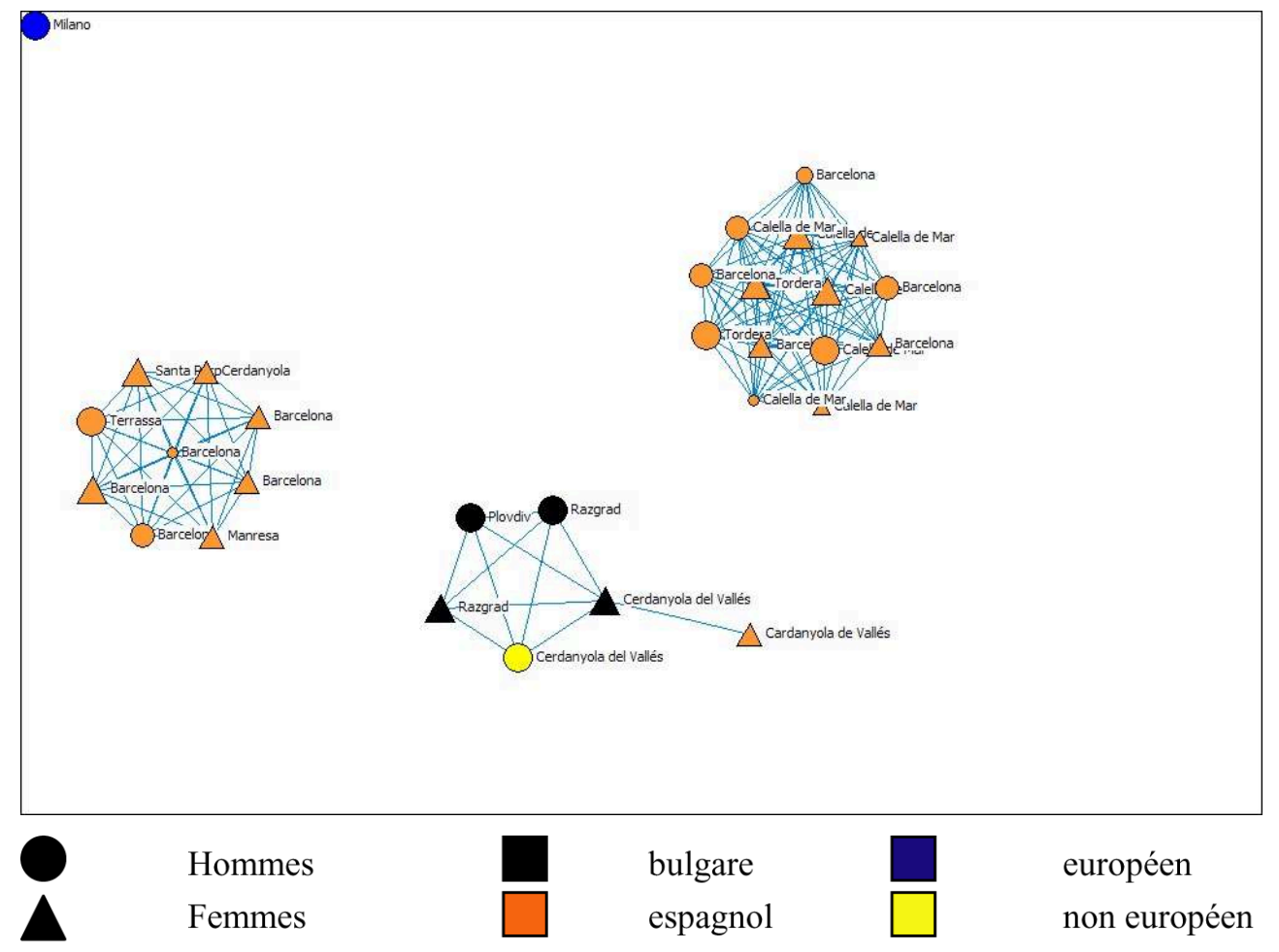

\section{Quelques regards sur les modalités d'adaptation et l'identité des Bulgares en Catalogne}

Selon Bauman ${ }^{37}$, il y a deux types d'immigrants : ceux que l'on pourrait définir comme des individus "cosmopolites globaux » et ceux qui sont enracinés dans leur localité spécifique. Cette idée met en relation l'identité avec le type d'adaptation à la société d'accueil. Les travaux de Miranda Lubbers, Jose Luis Molina et Chris McCarty ${ }^{38}$ soulignent déjà ce fait : ceux qui font partie de réseaux sociaux hétérogènes ont en même temps une identité plus globalisée (le troisième cas que nous avons décrit) alors que ceux dont le réseau social est plus homogène (en contact avec des compatriotes sur le lieu de destination ou sur le lieu d'origine) ont une identité plus locale, et sont donc plus attachés à leur pays d'origine (premier cas décrit, fig. 1).

Il est vrai que dans les petites villes, les Bulgares manifestent plus nettement leur identité locale bulgare alors que dans les grandes villes, leurs identités sont plus globalisées - ils affirment être des "citoyens du monde", ou des "citoyens de l'univers ». Néanmoins, on a aussi constaté l'existence de deux nouvelles identités plus ambiguës, avec d'autres schémas d'adaptation.

1) D'une part, nous avons mis en évidence un "groupe pont", ou "groupe intermédiaire " (fig. 2), constitué de personnes dont le réseau social est peu transnational et en même temps très enraciné dans la société d'accueil, avec presque la même proportion de contacts bulgares et autochtones sur le lieu de destination, et aussi avec des contacts avec d'autres nationalités. Ce groupe se déclare bulgare mais seulement au niveau culturel : leur identité politique, se traduisant par leur nationalité, 
semble plus floue et ambiguë. Parmi ces gens, cette identité politique est ressentie comme séparée et aliénée de l'identité personnelle, laquelle est liée à "leur expérience de vie" et à l'environnement social dans lequel ilsont vécu. Ils ressentent un faible lien avec les entités politiques de leur pays, ce qui les fait parfois douter de leur identité politique associée à l'État bulgare. Dans ce cas, ils manifestent être à la fois des citoyens du monde et des Bulgares seulement au niveau culturel.

2) D'autres se meuvent dans des réseaux sociaux très homogènes (essentiellement composés de Bulgares) et assez transnationaux, ou avec bien peu de contacts locaux (il s'agit de réseaux sociaux qu'on classerait dans le premier cas) et qui sont, en même temps, très intégrés dans la société d'accueil et dans la vie sociale de la localité, avec une bonne connaissance de la langue du pays ainsi que de la langue et la culture régionales. Il s'agit de personnes qui ont vécu un changement culturel mais qui maintiennent dans leur vie de forts liens avec leur culture bulgare et leur ethnicité, c'est-à-dire qu'ils ne se sont pas assimilés à la culture espagnole et catalane bien qu'ils y soient bien adaptés. On pourrait dire que ce groupe de personnes maintient un schéma d'adaptation très transnational.

Les écarts de formation intellectuelle sont évidemment une variable qui peut influencer la structure des relations sociales de chaque individu. Par exemple, la possibilité de connaître la langue du pays est un facteur qui facilite la socialisation dans la société d'accueil. Il s'agit d'une variable qui peut avoir une influence lors de la construction des relations interpersonnelles. De la même façon, les Bulgares qui ne connaissent pas la langue (espagnole ou catalane) et n'ont pas un niveau de formation intellectuelle élevé cherchent habituellement à se lier avec des compatriotes qui pourront les aider et faciliter leur séjour en Catalogne.

41 Dans ce sens, nous avons examiné les différentes variables expliquant la configuration de réseaux personnels homogènes qui ne sont pas nécessairement contradictoires. Parmi ces variables, on trouve le sentiment d'appartenance et/ou l'identification ethnique, mais aussi la façon d'entrer dans les pays de destination (c'est-à-dire la caractéristique de la chaîne migratoire à travers laquelle on a accédé au pays d'accueil); ou encore, les ressources sociales dont on dispose au début et que l'on maintient, c'est-à-dire les contacts avec d'autres compatriotes, qui peuvent à leur tour aider à conserver cette identification ethnique ${ }^{39}$.

42 Ainsi, on pourrait dire qu'il y a des Bulgares qui sont bien intégrés dans la sphère publique (ils parlent la langue régionale, ont des connaissances parmi les personnes locales et sont bien intégrés dans le circuit d'embauche local par exemple) tout en conservant, dans la sphère privée, un cercle de relations qui est très bulgare et transnational (comme nous pouvons le voir dans la composition de leur réseau de contacts personnels). Cela nous amène à penser que les caractéristiques des réseaux personnels peuvent être expliquées par le type d'adaptation au contexte local mais aussi par les caractéristiques de la chaîne migratoire et par les environnements sociaux les plus immédiats. Nous pourrions suggérer que ces derniers sont des immigrants qui maintiennent une certaine dualité d'adaptation, placés entre deux mondes, un monde bulgare et un autre espagnol.

Leur identité est aussi ambiguë : ils se déclarent Catalans, Espagnols ou Bulgares selon les circonstances, ce qui correspond bien au nouveau paradigme dutransnationalisme, selon lequel le processus d'intégration de l'immigrant ne contredit pas nécessairement le maintien - et même le renforcement - de liens avec la société d'origine. Pour 
beaucoup d'auteurs, ce nouveau paradigme permet de mieux comprendre les manières de vivre contemporaines des immigrants, entre « ici » et « là-bas ». À l'heure actuelle, la plupart des chercheurs reconnaissent que tous les immigrants ne développent pas nécessairement des liens transnationaux ${ }^{40}$. Ils reconnaissent aussi que parmi ceux qui les développent, le transnationalisme peut impliquer seulement une sphère de leur existence, et pas nécessairement la sphère la plus centrale et la plus significative. De la même manière, le développement de processus transnationaux ne signifie pas que les forces d'assimilation dans la société d'accueil aient disparu ou qu'elles se soient affaiblies. Il est clair que l'intégration des immigrants ne signifie pas leur assimilation dans la société d'accueil, contrairement à ce qu'affirmait Berry ${ }^{41}$.

Par ailleurs, comme nous l'avons déjà dit, il y a des migrants bulgares avec des réseaux personnels très hétérogènes. Ceux-là ont vécu un important changement culturel, tandis que dans la sphère privée ils maintiennent des relations très étroites avec quelques rares familles bulgares dans le pays de destination, avec qui ils partagent des célébrations et traditions de la Bulgarie (par exemple, la Pâque bulgare). En revanche, les personnes présentant ces types de réseaux sociaux ont rarement des contacts dans le pays d'origine, c'est-à-dire qu'ils ne s'inscrivent pas dans un schéma très transnational (fig.2).

Dans les grandes villes comme à Barcelone, les Bulgares maintiennent leurs propres traditions et d'autres aspects culturels dans un nombre réduit de cercles de relation et encore moins fréquemment au sein de certaines familles. Cela peut être expliqué par le fait qu'à Barcelone le contact avec d'autres compatriotes est plus difficile, en raison de la distribution des quartiers. Ensuite, les rares initiatives visant à maintenir leurs propres célébrations se produisent dans le cadre de maisons de quartier ou d'associations qui ne sont toutefois pas très nombreuses.

Comme conclusion on peut dire qu'il s'agit finalement plutôt d'une question d'identification, comme le suggèrent Brubacker et Cooper. L'idée d'identification se réfère au caractère développemental du phénomène plutôt qu'au caractère plus rigide du concept. En suivant cette perspective, on pourrait dire que l'identité correspond au processus d'identification qui est toujours relationnelle, circonstancielle et que l'on négocie continuellement en fonction de la situation.

Ainsi, Maya nous explique que: "mais quand tu es toute seule dehors...eh bien t'as envie de célébrer Noël comme je le fais en Bulgarie, en peignant des œufs pour Pâques... et ce sont des choses comme ça qui, finalement, font que tu t'identifies comme Bulgare ». Et Milena: « on se retrouve pour voir des choses de la Bulgarie et parce que tu t'identifies ".

C'est-à-dire que fondamentalement, c'est la culture - simplifiée dans les célébrations, les traditions et les festivités - qui fait que les Bulgares s'identifient en tant que tels.

\section{Les Bulgares dans la représentation mentale des Espagnols}

Selon la façon dont ils se sont établis et leur emprise sur le territoire, les Bulgares jouissent d'images diverses. Ainsi, leur visibilité et/ou invisibilité est un aspect très présent et qui est peut-être aussi dû à l'attitude adoptée par les migrants ou encore à la connaissance que les locaux ont de ces derniers. Ainsi, tout comme Dana Diminescu qui 
définit les migrants roumains comme "visibles mais peu nombreux", Soultanova définit les migrants bulgares comme « invisibles mais nombreux $»^{42}$. en chiffres absolus, surtout du point de vue des pays de destination (800 000 habitent et travaillent à l'étranger), ce chiffre représente entre 5 et $9 \%$ de la population de la Bulgarie, et $20 \%$ de la population bulgare recevrait une aide directe ou indirecte des migrants. Par ailleurs, à la différence des Roumains, les Bulgares ne sont guère présents dans les médias des pays de destination. La presse ne leur ménage guère de place, à l'exception de quelques rares scandales souvent liés à des affaires de prostitution, de crime organisé ou autres affaires maffieuses; on note aussi quelques nouvelles concernant surtout les compétitions sportives ou la vie culturelle ${ }^{43}$. Par contre, Soultanova ${ }^{44}$ signale que le phénomène de la migration des Bulgares à l'étranger est très présent en Bulgarie même: la presse nationale regorge d'articles concernant l'émigration bulgare. L'émigration est considérée comme la cause principale du déclin démographique, l'émigration irrégulière «ternissant l'image de la Bulgarie » et entravant l'intégration européenne du pays.

51 Si l'on compare les données statistiques de population migrante en Espagne, on peut conclure que les Roumains sont plus visibles parce qu'ils sont plus nombreux et que les Bulgares sont moins visibles parce qu'ils sont moins nombreux. Par contre, on sait que les Rroms roumains en Catalogne sont moins nombreux que les Roumains mais par contre plus visibles ${ }^{45}$ que les Roumains et les Bulgares dans leur ensemble! Comment expliquer ce phénomène? Nous partons de l'hypothèse selon laquelle il s'agit d'une question ethnique, mais aussi d'établissement et de présence territoriale. Les Rroms roumains sont toujours établis en communautés, et leur présence est plus visible bien qu'ils ne soient pas très nombreux. En partant de cette idée, nous n'avons pas voulu rapporter la plus ou moins grande visibilité du groupe bulgare à son poids démographique ${ }^{46}$ en Catalogne, mais plutôt aux caractéristiques de leur établissement et à l'existence ou à l'inexistence de communautés, ainsi qu'aux caractéristiques de leurs réseaux sociaux et d'autres variables - comme les types de relation que maintiennent les Bulgares avec les autochtones.

Ce que nous a dit une informatrice, qui résidait auparavant à Madrid, vient éclairer notre choix : «Je sens qu'ici, en Catalogne, en général les Bulgares sont très invisibles. Je sais bien qu'il y en a beaucoup mais comment est-ce que je peux les voir? Parce qu'à Madrid chaque fois que je marche dans la rue, j'entends parler bulgare et je rencontre toujours des Bulgares dans des lieux très différents, et dans différents itinéraires si je marche dans la rue (...). Je crois que nous sommes très invisibles en Catalogne, et un centre pourrait aider. Je pense que c'est parce que ceux qui sont ici sont peut-être arrvés il y a longtemps et sont mieux intégrés. Je ne sais pas, hein! Cela pourrait être une possibilité. Et Madrid est la capitale... elle attire toujours plus dans l'imagination des gens, des étrangers, même ici, en Catalogne, je pense qu'il y a plus de travail. Je pense que les gens vont à Madrid parce que c'est une grande ville et que c'est la capitale et qu'il y aurait là-bas plus de travail, ou pour des contacts $»^{47}$.

L'invisibilité ne semble pas être spécialement liée à la plus ou moins grande présence de Bulgares, bien que si l'on regarde les statistiques, on voit que la présence des Bulgares dans la communauté de Madrid est très supérieure à celle qui vit dans la communauté de Catalogne. 

catalane. D'un autre côté, l'invisibilité est aussi un aspect considéré comme une conséquence $\mathrm{du}$ caractère inhérent au peuple bulgare: "Nous sommes très individualistes, je ne sais pas comment te l'expliquer. Ceci c'est mon impression, que nous les Bulgares nous ne sommes pas très communautaires, surtout quand on est à l'étranger, nous sommes de mentalité plus indépendante $\aleph^{48}$, nous révélait une informatrice. Un autre informateur commente que «non, je ne crois pas que nous, les Bulgares, nous soyons ce type de personnes qui vont dans les associations, ce n'est pas typique, nous sommes plus individuels $»^{49}$. Par ailleurs, le peuple bulgare est souvent cité comme un peuple relativement récent, qui n'a existé qu'à partir de son émancipation de l'Empire Ottoman (1878), situé au carrefour de l'Occident et de l'Orient, et a connu beaucoup de cultures très différentes et indépendantes les unes et des autres tout en cohabitant ensemble.

En fait, ce qui est très révélateur n'est pas tant l'existence réduite d'associations bulgares (il y en a 14 dans tout l'État espagnol) mais la participation presque nulle des Bulgares à ces associations. Monica Ibañez ${ }^{50}$ attribue ce phénomène au faible activisme politique de ces associations mais aussi à un signe post-socialiste: «le communisme combattait toutes les connexions non institutionnalisées parmi la population bulgare $»^{51}$. D'ailleurs, on ignore jusqu'à quel point les minorités ethniques bulgares 
sont représentées dans ces associations qui doivent plutôt créer leurs propres réseaux et voies de manifestation particuliers.

Les associations étudiées par Ibañez ont un caractère transnational parce qu'elles rassemblent des individus et des groupes sociaux de plus d'un État et parce que leurs membres se perçoivent eux-mêmes comme membres de deux communautés politiques, sociales et culturelles. Pour autant, l'objectif de la plupart de ces associations est de maintenir des "vies transnationales", dans la mesure où ces individus appartiennent autant au groupe d'origine qu'au groupe de destination. Un autre des objectifs est de promouvoir la culture bulgare en même temps que la culture espagnole. Une association peut être comprise comme une plate-forme de rencontre de migrants en situation de vulnérabilité et/ou de minorité et peu représentés. Dans ce sens, une association peut aider à s'adapter à la société d'accueil. Parmi les migrants qui soutiennent la création d'associations bulgares, on jette un certain discrédit sur celles qui ont choisi de faire disparaître ou de rendre invisible leur culture d'origine dans le but de mieux s'adapter à la société d'accueil.

Dans ce sens, au cours des interviews, les Bulgares déclarent que la création d'associations, toujours adossée à l'idée d'« intégration ", se révèle à double tranchant. 1) D'une part, une association permet d'exprimer, chez certains, la volonté de ne pas s'« intégrer » et prélude à la constitution de « ghettos». C'est la raison pour laquelle certains informateurs ont déclaré que, malgré leur désir de monter une association, ils croyaient que ce ne serait pas bien considéré par la société d'accueil, bien qu'ils ne pensent pas vraiment que cela pourrait être contradictoire avec leur intégration. Ils pensent également qu'une telle initiative ne serait pas soutenue par tous les Bulgares. La discrétion est, comme nous l'avons déjà dit, l'une des caractéristiques des Bulgares qui, à plus d'une occasion, se comparent avec la communauté marocaine, les Marocains étant beaucoup plus nombreux, visibles et représentés dans la sphère publique, mais sont également soumis à un degré plus élevé de rejet de la part de la société d'accueil.

2) D'autre part, une association peut être considérée comme un lieu de réunion pour des migrants en situation de vulnérabilité puisqu'ils sont en minorité ou sousreprésentés dans la société d'accueil. Les bulgares pourraient y trouver l'aide nécessaire pour s'adapter à la société d'accueil sans quitter leur culture et leur identité d'origine. Néanmoins, bien que nous percevions un désintérêt en ce qui concerne la participation à des associations d'immigrants bulgares, en revanche, il existe une participation assez importante dans d'autres entités et associations comme par exemple l'UNESCO, Greenpeace, Oxfam, l'Église orthodoxe bulgare, et quelques centres sportifs locaux (33\% des interviewés participent à la vie associative, $67 \%$ n'y participent pas).

62 D'autres aspects interviennent pour freiner la vie d'associations de migrants bulgares: "méfiance", "rivalité » entre compatriotes pour accéder aux ressources sociales, " peur » d'être pris au piège dans un système de faveurs et contre-faveurs typique de l'entraide entre compatriotes faisant usage de leur capital social pour faciliter leur entrée, leur installation et leur bien-être dans la société d'accueil. La perversion de ces relations est une crainte souvent exprimée par les Bulgares; elle les motive à être prudents et à sélectionner les compatriotes qu'ils ajoutent à leur réseau social. Un informateur l'a exprimé ainsi : " Tous sont très amis tant que c'est nécessaire ».52

63 Par ailleurs, les Bulgares construisent leur image à partir de celle que leur renvoient les autochtones. Nos informateurs prétendent que l'image des Bulgares est biaisée parce 
qu'on les associe au groupe immigrant dominant du sud-est de l'Europe, les Roumains, qui sont pour leur part bien présents et visibles dans la population d'accueil. Réaffirment par là l'image négative de ce groupe, ils s'en distinguent en exposant l'histoire de leur pays mais aussi leurs us et coutumes. Une informatrice manifeste ainsi son auto image par opposition aux Roumains : «Nous ne sommes pas roumains, nous sommes différents. L'auteur du premier ordinateur est bulgare, plus de 700000 ingénieurs informaticiens bulgares travaillent en Italie où ils font des programmes, c'est une nation intelligente. Mais ici, quand on dit Bulgare : "ah! La Roumanie", mais ça n'a rien à voir... Il y a beaucoup de voleurs roumains, mais ce ne sont pas des Bulgares, mais ils se mélangent toujours... les gens doivent faire attention... (...). Du fait que la Bulgarie était avant comme l'une des 16 républiques [de la Russie], rien à voir avec la Roumanie. Les Russes écrivent en cyrillique, [un alphabet d'origine] bulgare. Cela veut dire que ce pays a beaucoup de choses dans le monde (...). On n'a rien à voir avec les Roumains. En Europe, en Angleterre, en Hollande, en Belgique, tout le monde respecte les Bulgares mais pas ici... En France et en Italie non plus. Peut être qu'ils ne savent pas ... ne connaissent pas... Ce sont des travailleurs, les Bulgares.... ${ }^{53}$. Cette image du Bulgare est récurrente chez les informateurs. Non seulement l'image des Bulgares est négative parce qu'elle est associée à celle des Roumains, mais ils en deviennent délinquants, criminels, voleurs : "Quand tu montres ta carte d'identité et que tu dis que tu es Bulgare, la façon de se comporter est différente. Les gens te regardent de travers et ils pensent que tu es un brigand. Les Bulgares ont une mauvaise réputation. Je veux vivre normalement $\aleph^{54}$. Autre aspect générateur d'images négatives, le fait d'avoir ou non des papiers - et, dans une autre mesure, d'être Européen ou non. Devenir Européen a été vécu par de nombreux Bulgares comme une ouverture à l'égalité de droits en même temps que la possibilité de démythifier l'image des Bulgares-délinquants. En bref, on pourrait dire que dans ce nouveau contexte de mobilité que représente l'Europe, espace commun de mobilités transnationales et de rencontres avec l'autre lui aussi européen, ce ne sont pas les frontières et la conscience de groupe qui provoquent des expériences identitaires, comme le disait Frederik Barth ${ }^{55}$, mais plutôt, comme le soutien Ivaylo Ditchev, ce sont les pratiques de mobilités, les rencontres avec le rival, la lutte pour les ressources qui produisent des différences et qui peuvent se traduire par des expériences identitaires différentes.

\section{Conclusion}

Les Bulgares ont un comportement relationnel très varié, qui entraîne différents processus de changement culturel. En règle générale, ils sont vite absorbés par la société d'accueil, ne manifestant leur culture et leur identité que dans la sphère privée ou dans le cadre de relations dyadiques (ami d'ami, parent d'ami, etc.) à travers lesquelles ils construisent des réseaux très intimes et sélectifs à partir d'affinités culturelle et identitaire. 


\section{NOTES}

1. Yordan, Roses, $02 / 07 / 2008$.

2. Daniela, Barcelone, 25/06/2008.

3. Draganova-Madelaine (Evguénia), La Bulgarie face à l'Europe. De la transition à l'intégration, L'Harmattan : Paris, 2004

4. Giordano (Christian), Kostova (Dobrinka), «The social production of mistrust », in Hann (C.M.), ed., Postsocialism, ideals, ideologies and practices in Eurasia, Routledge : London, 2000.

5. Elchinova (Magdalena), «Migration Studies in Bulgaria: Scope, Experiences and Developments », Anthropological Journal of European Cultures, 18 (2), 2009.

6. Il faut préciser que les Bulgares sont présents aux États-Unis dès les années 1870, surtout à Chicago. Il s'agissait d'étudiants envoyés par les missionnaires américains protestants. La plupart rentrèrent chez eux; ceux qui restèrent aux États-Unis jetèrent les fondations d'une communauté qui s'est développée jusqu'à aujourd'hui.

7. Veiga(Francisco), La trampa Balcánica, Barcelona : Grijalbo, 1995.

8. Markova (Eugenia), «Trabajadores búlgaros en Madrid. Situación legal y actuación en el mercado de trabajo ", Revista CIDOB d'Afers Internacionals, (84), 2008.

9. L'espace méditerranéen est particulièrement attrayant pour les émigrants bulgares - surtout à partir 2003, quand $40 \%$ d'entre eux voulaient partir pour plus d'un an à l'étranger. Mais il faut comprendre moins la Grèce et l'Italie, proches de la Bulgarie, que l'Espagne ( $18 \%$ des candidats à l'émigration à court terme, c'ést-à-dire qui veulent rester moins d'un an à l'étranger, et $12 \%$ des émigrants qui veulent s'établir définitivement à l'étranger). Voir Mintchev (Vesselin), Boshnakov (Venelin), Kaltchev (Jordan), Goev (Valentin), «Who is Leaving? Potential Emigration from Bulgaria in the Beginning of the XXI Century ", 2003 (à l'adresse: http:// www.cerge-ei.cz/pdf/gdn/RRCIII_28_paper_01.pdf).

10. Observatorio Permanente de la Inmigración (Ministerio de Trabajo y Asuntos Sociales, Secretaría de Estado de Inmigración y Emigración), « Año 2007, tercer trimestre - Extranjeros con certificado de registro o tarjeta de residencia en vigor ", http://www.mtas.es

11. INE (Instituto Nacional de Estadística), 2007.

12. Observatorio Permanente de la Inmigración, « Año 2008, Boletín Estadístico de Extranjería e Inmigración. 17 agosto 2008 », http://extranjeros.mtin.es

13. Moratoire annexé au Décret royal 178/2003 du 14 février, sur l'entrée et le séjour en Espagne des nationaux appartenant aux États membres de la UE

14. Communication personnelle de Carme Pozo, chargée de recrutement de main-d'œuvre étrangère (CECOT/29.02.2008).

15. Voir Gomez (Sílvia), Hoecksma (Sarah), Molina (Jose Luis), «The personal networks of Small-scale Bulgarian Enterpreneurships in Catalonia », in Chavdarova (Tanya), Slavoya (Petya), Stoeva (Svetla), eds., Markets as Networks, Sofia : University of Sofia, 2010.

16. González (María Pilar), Aguilera (María José), «La procedencia, a escala local, de los inmigrantes extranjeros en España a finales del siglo XX », Espacio, Tiempo y Forma, 4 (11), 1998.

17. Gómez(Sílvia), Molina (Jose Luis), Hoeksma (Sarah), "Patterns of Social Networks of Bulgarian Migrants in Catalonia: Two study cases (Barcelona and Roses) »,Paper presented at the International Conference AnthropoEast2008. The Europeisation of Balkans. The Balkanisation of Europe, $3^{\text {rd }}$ Edition, 2008.

18. Ibañez (Maria Pilar), « Nation building within the European Union : Reframing Bulgarian National Identity from abroad», in Marushiakova (Elena), ed., Dynamics of National Identity and Transnational Identities in the Process of European Integration, Newcastle: Cambridge Scholars Publishing, 2008; Deneva (Neda), Circumventing the category: the Double-naming Strategy of 
Bulgarian Muslim Migrants in Spain, Prague: Multicultural Centre Prague, 2008. Voir http:// www.migrationonline.cz/.

19. Markova (Eugenia), art.cit.

20. Il faut prendre en compte que le territoire espagnol se divise en différéntes communautés qui, chacune, a son propre système administratif. Chaque communauté comporte plusieurs villes.

21. Anuario estadístico. Tabla 13.1. Extranjeros con certificado de registro o tarjeta de residencia en vigor según provincia y nacionalidad 30/09/2008.

22. Deneva (Neda), op.cit.

23. Markova (Eugenia), art.cit.

24. Deneva (Neda), op.cit.

25. Slavkova (Magdalena), «Being Gypsy in Europe. The Case of Bulgarian Roma Workers in Spain », Balkanologie, 11 (1-2), 2008. Voir http://balkanologie.revues.org/.

26. De toute façon, il paraît que l'immigration familiale représente la moitié des migrations permanentes en Europe, migrations matrimoniales inclus. La plupart accèdent au marché de travail - voir De Wenden (Wihtol), Benoît-Guyod (Madeleine), Atlas Mondial des migrations, Paris : Autrement, 2009.

27. González (María Pilar), Aguilera (María José), art.cit.

28. Partir à l'étranger pour étudier est un type de mobilité déjà existant au début du XIX ème siècle, en raison des aléas politiques et du retard pris dans la construction d'universités et d'écoles secondaires (voir Soultanova (Ralitza), «Les migrations multiples de la population bulgare ", actes du colloque La France et les migrants des Balkans : un état des lieux, 20 janvier 2005, Paris, Courrier des Balkans, à l'adresse: http://balkans.courriers.info/IMG/rtf/ Les_migrations_multiples_de_la_population_bulgare.rtf).

29. López (Oscar), Sàez (Meritxell), « Forced Mobilities, Marginal Positions and Access to Basic Rights. Roma Migrants and Local Politics in Barcelona Metropolitan Area », in Fosztó (László), ed., Two Decades of Scholarship: Romani Studies in Romania (1989 - 2009), Cluj-Napoca : Romanian Institute for Research on National Minorities, 2010.

30. Marushiakova (Elena), Popov (Vesselin), « Les migrations des Roms balkaniques en Europe occidentale: mobilités passées et présentes », Balkanologie, 11 (1-2), 2008 ; Ragaru (Nadège), «Imaginaires et itinéraires migratoires bulgares en Europe. Une introduction », Balkanologie, 11 (1-2), 2008. Voir http://balkanologie.revues.org/.

31. Selon Marushiakova \& Popov (Marushiakova (Elena), Popov (Vesselin), art.cit.), il semble que ceci pourrait s'expliquer par la forte pression de l'État communiste bulgare pour sédentariser les roms, en échange de facilités économiques. Ce groupe s'est principalement installé dans les centre-ville, en se mêlant à la population. Dans la majorité des cas, ils se sont donc intégrés en tant que Bulgares non roms. À l'inverse, à Sliven, il existe un quartier spécifiquement rom, d'où proviennent les hommes rom musiciens qui se partagent entre le quartier de Barceloneta (Barcelona) et celui de l'Espoir (Sliven).

32. Marushiakova (Elena), Popov (Vesselin), art.cit.

33. Le gurbet est une forme traditionnelle de mobilité professionnelle qui se pratiquait pendant l'empire ottoman: des groupes de population - de quelque ethnie que ce soit - étaient sélectionnés pour partir travailler dans d'autres pays du même Empire (dans les Balkans) pendant le temps que durait l'activité économique (Voir ibid.) Le gurbet transnational, phénomène récent, est appelé gurbetchii (Voir Slavkova (Magdalena), art.cit.).

34. Soultanova distingue différents gurbet-s : système de circulation pendulaire, mais aussi seminomadique ou occasionnel, comprenant dans ce cas une mobilité entre deux lieux ainsi qu'une double résidence (Soultanova (Ralitza), art.cit).

35. Communication personnelle de Kim Arnal, directeur d'une compagnie de musique romani de Roms bulgares. 
36. AAVV, Gitanos procedents de l'Europa de l'Est a Catalunya, Barcelona : Dep. de Benestar i Familia, 2006 ; PeetersGrietens (Koen), Entre tenebres. Gitanos immigrants: els rrom de l'Est d'Europa a Barcelona $i$ el seu accés a l'educació, Barcelona: Ed Fundació Jaume Bofill, 2005 ; Vada (Maximiliano), Dimitrova (Luba), Ojos negros. Una historia que nunca muere, Lada Film, 2005 37. Bauman (Zygmunt), La globalización : consecuencias humanas, México DF : FCE, 1999.

38. Lubbers (Miranda), Molina (Jose Luis), Mccarty (Christopher), « Personal Networks and Ethnic Identifications : The Case of Migrants in Spain », International Sociology, 22 (6), 2007.

39. L'étude de Lubbers, Molina et McCarthy (ibid.) suggère que l'identification ethnique influence les réseaux sociaux et inversement, sans qu'on sache dans quel sens le processus s'opère.

40. Castles (Stephen), «Transnacional Communities : a new form of social relations Ander conditions of grobalization? ", in Reitz (Jeffrey), ed., Host Societies and the Reception of Immigrants, San Diego: Universitat de Califòrnia, 2007; Portes (Alejandro), «Un diálogo norte-sur : el progreso de la teoría en el estudio de la migración internacional y sus implicaciones ", in Ariza (Marina), Portes (Alejandro), eds., El país transnacional : migración mexicana y cambio social a través de la frontera, Mèxic: Universidad Nacional Autónoma de México / Instituto de Investigaciones Sociales, 2007 ; Landolt (Patricia), "The Transnacional Geographies of Immigrant Politics: Insight from a Comparative Study of Migrant Grassroots Organizing ", The Sociological Quarterly, 49, 2008.

41. Berry (J. W.), «Inmigration, Acculturarion and Adaptation », Applied Psychology, 46 (1), 1997.

42. Soultanova (Ralitza), art.cit.; Diminescu (Dana), Visibles mais peux nombreux. Les circulations migratoires roumaines, Paris : Éditions de la Maison des Sciences de l'Homme, 2003.

43. Nous sommes en train d'analyser presque 1800 encarts de presse concernant les migrants bulgares et les migrants roumains publiées entre 2008 et 2010 . Il s'agit d'un travail qui sera développé au sein du groupe de recherche Egoredes de l'Université Autonome de Barcelone.

44. Soultanova (Ralitza), art.cit.

45. En Catalogne, il y a entre 3000 et 4000 Roms, mais ces données changent de mois en mois à cause de la grande mobilité de cette population. Malgré tout, on peut affirmer qu'ils ne représentent pas plus de $5 \%$ du total de la population roumaine installée en Catalogne. López (Oscar), Sàez (Meritxell), art.cit.

46. La grande concentration de Bulgares dans les villes de Getafe et de Parla (région de Madrid), ainsi que la création d'importantes communautés, permet une plus grande visibilité de ce groupe.

47. Maya, Barcelone, 23/08/2008.

48. Yanina, Barcelona, $20 / 06 / 2008$

49. Boyan, Barcelona, 02/07/2008

50. Ibañez (Mónica), art.cit.

51. Iliycheva (Maria), «Philanthrophy and Social Capital: Theoretical Aspects and Empirical Examples from Bulgaria », Tischner Debates Onlinewww.erazm.uW.edu.pl//www.iwm.at/index, 2005.

52. Emilia, Roses, 30/06/2008

53. Yotko, Barcelona, 07/08/08

54. Yordan, Roses, 02/07/08

55. Barth (Frederick), Ethnic Groups and Boundaries: The social Organisation of Cultural Difference, Boston : Little Brown, 1969. 


\section{RÉSUMÉS}

Cette étude analyse les processus d'établissement, l'adaptation et la dynamique des communautés de migrants bulgares en Espagne et, plus particulièrement, en Catalogne, à travers l'étude de leurs réseaux sociaux. L'article montre la manière dont la distribution des Bulgares sur le territoire espagnol ainsi que leurs modalités d'adaptation sont caractérisées par le type de chaîne migratoire et par les réseaux sociaux. Ceux-ci sont surtout fondés sur la famille en tant qu'unité sociale de base, et non sur la communauté ethnique et/ou religieuse. La base la plus importante en est le cercle fermé des parents. Se construisent des réseaux familiaux et amicaux indépendants les uns des autres, ce qui se traduit par le fait que des membres de la même famille, ou des amis et voisins s'établissent dans des localités différentes et de façon très dispersée. D'autres variables interviennent en outre: le niveau de formation, l'âge, la structure de la population dans laquelle ils s'incorporent, la connaissance de la langue et l'acquisition de ressources sociales, ainsi que les schémas de relation qui existent dans chaque localité. Tout cela se traduit par des différences entre les migrants bulgares, que ce soit en ce qui concerne les modalités d'adaptation, les formes basiques de changement culturel ou l'identification ethnique. La différence dans le type d'établissement et de présence territoriale des Bulgares influence aussi les images qui sont construites sur ce collectif, ainsi que leur visibilité et/ou invisibilité dans la société d'accueil. Le degré de participation des citoyens dans la vie civile, lui aussi très significatif quant aux modalités d'adaptation, est examiné à travers les associations bulgares.

This study analyzes the process of establishing, adaptation and the dynamism of communities of Bulgarian migrants in Spain and especially in Catalonia through the study of their social networks. The article shows how the distribution of Bulgarians in Spain and the modalities of adaptation are characterized by the type of migratory chain and the features of their social networks. Mostly these social networks are based on kinship ties -and not on the ethnic or religious community-, in which the most important are those related with the closed circle of parents, although configurations of friends and neighbors are also present. Other variables for explaining the types of adaptation are educational attainment, age structure of the population from which they are incorporated, the knowledge of the language, the acquisition of social resources, and the pattern of relationships which exist in each locality. All this leads to differences among the Bulgarian migrants, whether in changes in some cultural traits or in their ethnic identification. The pattern of territorial distribution influences the images built by the host society upon the collective and their visibility-invisibility. Finally, the degree of involvement in civil society is analyzed through the role developed by Bulgarian associations.

\section{INDEX}

Index géographique : Bulgarie, Espagne, Europe, Roumanie

Keywords : Bulgarian, immigration, migration chains, social networks

Mots-clés : Bulgares, chaînes migratoires, immigration, réseaux sociaux 


\section{AUTEURS}

\section{SÍLVIA GÓMEZ-MESTRES}

Universitat Autònoma de Barcelona, Laboratori de xarxes personals i Comunitats. Facultat de lletres, Edifici-E, 08193 Bellaterra (Spain).

silvia.gomez@uab.cat/anxovetes@yahoo.es

\section{JOSE LUIS MOLINA}

Universitat Autònoma de Barcelona, Laboratori de xarxes personals i Comunitats. Facultat de lletres, Edifici-E, 08193 Bellaterra (Spain).

joseluis.molina@uab.es 